\title{
ACCESO Y PERMANENCIA A LA EDUCACIÓN SUPERIOR DE MUJERES INDÍGENAS MAYANGNAS, URACCAN LAS MINAS, 2009 - 2010
}

Sorayda del Carmen Herrera Siles ${ }^{[1]}$

\section{Resumen}

Se ha descrito el acceso y permanencia en la Educación Superior de mujeres indígenas mayangnas en el Recinto URACCAN Las Minas, período 2000-2009, en las comunidades de Wasakín y el Cuarto Territorio Indígena Mayangna (Mayangna Sauni Arunka), ubicadas en los municipios de Rosita y Bonanza, Región Autónoma del Atlántico Norte (RAAN). Se realizó en el marco en una perspectiva cualitativa, a partir del enfoque etnográfico con el apoyo de la observación y la entrevista.

La diferencia cultural y la pobreza que implica la falta de recursos monetarios, han sido los principales obstáculos que limita a las jóvenes indígenas al acceso y permanencia de la Educación Superior. Las mujeres indígenas no tienen la posibilidad de ejercer plenamente sus derechos ciudadanos en igualdad de condiciones, carecen de oportunidades para desarrollar sus capacidades y talentos.

Los embarazos han sido la causa principal por la cual la mayoría de las mujeres indígenas abandonan sus estudios superiores. Se destaca asimismo, que solamente la URACCAN hace presencia en el territorio Mayangna Sauni Arunka y la comunidad de Wasakín para promover el acceso y la permanencia de las mujeres en la Educación Superior por medio de un sistema de becas.

Palabras clave: Educación Superior, acceso y permanencia, dificultades, mujeres indígenas.

\section{Summary}

We have described the access and permanence to Higher Education of Mayangnas indigenous women in the URACCAN-Mines Campus, during the period 2000-2009, in Wasakin communities and the Fourth Mayangna Indigenous Territory (Mayangna Sauni Arunka), located in the municipalities of Rosita and Bonanza, North Atlantic Autonomous Region (RAAN). This study was performed using a qualitative perspective, based upon an ethnographic approach supported with observations and interviews.

\footnotetext{
${ }^{[1]}$ Máster en Docencia Universitaria. Coordinadora proyecto FOCUS SAIH, CEIMM, URACCAN Las Minas. barriodolores77@yahoo.com
} 
Cultural difference and poverty that implies a lack of monetary resources in cash have been the main obstacles limiting the indigenous youth to access and remain in Higher Education. Indigenous women are unable to exercise fully their citizen rights in equal conditions, and they lack of opportunities to develop their skills and talents.

Pregnancies have been the main cause why most indigenous women leave college. It's important to highlight, that only URACCAN has presence in the Mayangna Sauni Arunka territory and in the community of Wasakin to promote access and retention of women to higher education through a scholarship system.

Keywords: Higher Education, access and permanence, difficulties, indigenous women.

\section{Introducción}

Para consolidar una sociedad con equidad y justicia es fundamental analizar y trabajar en la situación de género en todos los ámbitos y de manera especial en la Educación Superior por su rol fundamental para el desarrollo personal, político y social. En términos generales, cada vez más mujeres acceden a la Educación Superior en América Latina y llegan incluso a ser mayoría. Sin embargo, el acceso y permanencia en el sistema universitario de las mujeres indígenas y de los sectores populares son aún insuficientes. De hecho, los indígenas en general se topan con escollos de diversa índole que marcan asuntos étnicos, culturales y lingüísticos en la Educación Superior y el ámbito universitario en particular.

La igualdad de oportunidades para la mujer ha sido usada como un concepto paraguas, tanto en los organismos internacionales como por parte de los gobiernos que promueven este tipo de políticas y los especialistas en la temática de la mujer. El significado más común es que las mujeres y los hombres deben tener las mismas oportunidades en términos de derechos formales y de acceso a iguales beneficios en el plano: educativo, trabajo remunerado, estatus social y bienes económicos. Sin embargo, el concepto es ambiguo y se presta a distintas interpretaciones que, por otro lado, tienen consecuencias ulteriores en la definición de políticas en este campo.

El estudio sobre el acceso y permanencia en la Educación Superior de mujeres indígenas, Recinto URACCAN - Las Minas, permitirá analizar hasta qué punto se toman en cuenta las perspectivas étnicas y culturalmente diferenciadas de género en la Educación Superior, tanto a nivel institucional y político, como epistemológico. Este análisis servirá de base para poder proponer estrategias y acciones concretas desde una perspectiva intercultural de género, que permita superar las dificultades que tienen las mujeres indígenas mayangnas para acceder y permanecer en la Educación Superior. 
Se aborda con énfasis las diferencias entre los sexos, diferencias que serían categorías uniformes, opuestas y no cambiantes a lo largo del tiempo. Donde se sigue afirmando que las mujeres, por razones biológicas y/o culturales han desarrollado características de personalidad y modos de relación y conducta social que deberían ser revaluados como un componente fundamental de la experiencia humana, y eventualmente extendidos al conjunto de la comunidad.

Si bien, en las últimas dos décadas, los pueblos indígenas han venido logrando avances significativos en varios asuntos que competen a su calidad de vida. No obstante, estos avances son aún insuficientes, como parte de los efectos de siglos de discriminación.

En el caso de las mujeres indígenas el campo educativo es uno de los pocos ejemplos donde se puede hablar de "brecha traspasada". Aunque las mujeres indígenas han estado muy ligadas al proceso educativo. En muchos pueblos, son las mujeres quienes transmiten los saberes, las tradiciones, las formas de comportamiento, en definitiva la propia cultura. Sin embargo, cuando hay posibilidades de destinar recursos para la educación, éstos son dirigidos a los hombres.

\section{Revisión de Literatura}

El mundo de la educación cumple los dos requisitos básicos para que pueda constituirse en objeto de una ciencia. En primer lugar está constituido por un conjunto de prácticas, de maneras de proceder, de costumbres, que son realidades todas ellas exteriores e independientes de la voluntad individual. El segundo requisito es la homogeneidad de estas prácticas, las cuales, en el caso de la educación, consisten en la acción ejercida por la generación adulta sobre la joven (Feito, 2009: 1).

Según Luke (1999), en los últimos años, la teoría y la investigación feminista se han difundido de forma exponencial, por su diversidad de investigaciones tanto como de teorías, dejando claro que la identidad y las relaciones de género no pueden asimilarse ni elaborarse en el plano teórico en sus propios términos abstractos. Es decir, el sexo, el género o la feminidad deben estudiarse y elaborarse en su relación constitutiva con otras significaciones socioculturales, historias económicas y políticas, jerarquías y discursos (p. 17).

Como señala Alta Hooker, en el contexto de las Regiones Autónomas de la Costa Caribe nicaragüense, "Cuando tenemos pueblos con culturas diferentes, pueblos que priorizan su territorio, su lengua, su religión, su familia, su entorno, que luchan por su autodeterminación y que ven con respeto y veneración a sus ancestros; cuando los pueblos abordan la vida desde ese contexto que no concuerda con la visión de esa mayoría que ocupa los círculos de poder, entonces hay exclusión." Es necesario retomar el concepto de Educación Intercultural (Saballos, 2007: 5). 


\section{Dificultades que encuentran las mujeres indígenas para acceder a la educación}

\section{Acceso a la educación}

La educación entendida como un proceso de socialización y aprendizaje orientado al desarrollo integral de la persona, es un derecho humano fundamental y condición básica del desarrollo social de los pueblos, por lo tanto una obligación de todo Estado procurar una educación de calidad y el acceso equitativo de la ciudadanía, creando las condiciones necesarias para su cumplimiento.

Para Velázquez (2006), hablar sobre mujeres indígenas y educación en México puede parecer un tanto contradictorio, en la medida que la Educación Superior en México es un privilegio de muy pocas personas. La dificultad de acceso a la educación de las mujeres indígenas se vuelve un problema complejo porque entran en juego por lo menos cuatro elementos: primero, el sistema neoliberal ha profundizado el rezago educativo al no contemplar entre sus prioridades el apoyo a la educación; segundo, la marginación que los pueblos originarios han sufrido, al otorgarles la función de productores artesanales, de materia prima y folklor para consumo de la sociedad nacional, siempre, circunscritos al ámbito rural; tercero, la exclusión de los pueblos originarios del proyecto nacional, derivada de la ideología colonial impuesta desde la conquista y sustentada en el sometimiento de su cosmovisión a una cultura ajena; estos elementos en conjunto han contribuido a crear el escenario actual de extrema pobreza que viven los pueblos originarios de México, siendo las mujeres indígenas las más pobres entre los pobres (p. 2).

Otra fuerte razón que limita a la mujeres a la educación es la situación económica del entorno familiar que las lleva a realizar dobles y triples jornadas laborares, dado que la pobreza afecta en lo general a hombres y mujeres; pero, en particular a la mujer; quien se enfrenta a la misma al administrar la economía familiar. Cuenta con menos oportunidades de obtener un empleo para contribuir al sustento del hogar (Madrigal, et al. 2005 p. 187).

\section{Permanencia de las mujeres indígenas en la Educación Superior}

La proporción de indígenas que tienen acceso a la universidad, y que completan una educación de nivel superior, es mucho menor que la del resto de la población estudiantil. Reynaga (2008) señala que, son muchos los factores que limitan el acceso de las mujeres y particularmente de las mujeres indígenas a la Educación Superior. La mayor parte de estos se desprenden de las políticas educativas que no contemplan las diversas realidades socioculturales y lingüísticas del estudiantado, que inciden desde la educación básica o primaria, limitando el acceso, la permanencia y la conclusión 
satisfactoria en cada nivel; sin embargo, para acceder a la Educación Superior no basta sólo con la conclusión de los niveles previos, sino que requiere de la calidad y la pertinencia de la educación pre-universitaria, que les garantice una formación adecuada e integral, que fortalezca las identidades y autoestima estudiantil y les posibilite competir en igualdad de posibilidades y condiciones con cualquier estudiante de su nivel, lo cual no es así (p. 4).

En la Educación Superior para pueblos indígenas, dos temas adicionales merecen alguna reflexión. El primero está referido a las becas y el segundo al papel que en el proceso de construcción de una Educación Superior para o con indígenas ha tenido la cooperación internacional. En ambos casos estamos ante la importancia que cobra el factor del financiamiento en el apuntalamiento de la Educación Superior indígena. En general, mientras las becas van destinadas a asegurar el avance individual de aquellos estudiantes indígenas a los que estas favorecen, la cooperación internacional apoya el funcionamiento de programas alternativos que buscan dotarse de pertinencia cultural y relevancia social. No obstante, cabe señalar que, conscientes de esta última necesidad, a veces los programas de becas incluyen pequeños montos para el mejoramiento de la oferta educativa (López, et. al. 2009: 258).

Papel que han jugado las organizaciones locales que inciden en el desarrollo de las comunidades indígenas en la inserción de las mujeres adolescentes y jóvenes a la Educación Superior y su permanencia en ella. Las organizaciones de mujeres, de los derechos humanos y de pueblos indígenas han desarrollado diversas acciones para poner plenamente en vigencia el principio de igualdad de los derechos humanos en el derecho internacional. En consecuencia, la comunidad internacional está uniendo sus esfuerzos para integrar los derechos humanos de las mujeres y de los pueblos indígenas a los sistemas y mecanismos de protección de los derechos humanos.

En este proceso se logran ciertos avances, que reflejan una interpretación sensible en la intersección de género, etnia, y otras condiciones de derechos humanos, tales como: La Convención para la Prevención y Sanción de Delitos de Genocidio (1948), La Convención sobre los Derechos Políticos de la Mujer (1948), La Convención Interamericana sobre la Eliminación de todas las formas de Discriminación Racial (1965), El Convenio 169 sobre Pueblos Indígenas y Tribales de la Organización Internacional del Trabajo (OIT), La Convención sobre la Eliminación de todas las formas de Discriminación contra las Mujeres (1981), entre otras (Callamard, 2002. p. 5).

El Sistema Educativo Autonómico Regional (SEAR) tiene sus fundamentos jurídicos en base a la Constitución Política de Nicaragua y la Ley No. 28, resaltando en sus fundamentos la autonomía, la educación de los pueblos indígenas, comunidades étnicas y afrodescendientes, la equidad de género, los derechos de las mujeres, niñez y adolescencia, el fortalecimiento de la identidad étnica y lingüística, entre otros (Alemán, s/f: 12). 


\section{La institución universitaria}

Dado el alcance y el ritmo de las transformaciones, la sociedad cada vez tiende más a fundarse en el conocimiento, razón de que la Educación Superior forme hoy en día parte fundamental del desarrollo cultural, socioeconómico y ecológicamente sostenible de las personas, las comunidades y las naciones (UNESCO, 1998.) citado por Castillo \& Lee (2009: 31).

El principio metodológico del modelo pedagógico de URACCAN, es relacionar lo endógeno con lo exógeno tanto en procesos productivos como culturales, logrando con esto conocer y valorar los saberes occidentales pero también valorar y mejorar lo propio. La formación que ofrecen la universidad comunitaria está basada fundamentalmente en el principio de la interculturalidad que permite a docentes y estudiantes tener una relación horizontal con las comunidades permitiendo que la práctica académica sea un espacio de interrelación de confianza y esperanza entre los sujetos multiétnicos (Modelo pedagógico de URACCAN, 2004, p. 15)

\section{Políticas compensatorias o acciones afirmativas en la Educación Superior}

Pedroza \& Villalobos (2008: 7), señalan que las políticas compensatorias aplicadas en la Educación Superior se sustentan en la concepción de la justicia como equidad: compensar las desigualdades para el logro de las oportunidades y la obtención de los beneficios. Debemos precisar que no existe una sola concepción en la idea de compensar, ya que existen distintas finalidades desde posturas ideológicas diferentes, no es lo mismo proponerse la compensación desde una lógica social distributiva que desde la lógica racionalista del mercado.

Las políticas no sexistas subrayan la importancia de integrar a la currícula lo «femenino» de manera revalorizada; promover en las jóvenes el surgimiento de un sentimiento de solidaridad con su género y una base de confianza y autovaloración por el hecho de ser mujeres; reemplazar la organización escolar fundada en parámetros jerárquicos, competitivos y autoritarios por pautas y procedimientos basados en la cooperación, la democracia, la solidaridad y la equidad. Sobre este punto es ilustrativo que algunas autoras propongan la creación de una escuela «amigable para las niñas» (Bonder, 1994: 25).

\section{Materiales y métodos}

El estudio se enmarca en una perspectiva de investigación cualitativa, de carácter descriptiva y se desarrolló a partir del enfoque etnográfico. 
La población sujeta a estudio fueron las mujeres indígenas mayangnas que han accedido al sistema de becas como estudiantes en las diferentes carreras que se oferta en la URACCAN - Las Minas, en el período 2009 y el primer semestre del 2010 en las carreras de Administración de Empresas, Ciencias de la Educación con mención en Pedagogía, Español, Matemáticas, Biología, Ciencias Sociales con mención en Desarrollo Local, Ingeniería Agroforestal y Zootecnia e Informática Administrativa y las mujeres indígenas mayangnas que han completado estudios secundarios.

Se utilizó el método de la observación y la entrevista que son métodos considerados por excelencia en la investigación cualitativa.

\section{Resultado y discusión}

Dificultades que enfrentan las mujeres indígenas mayangnas para acceder y permanecer en la Educación Superior.

La oferta educativa en los territorios indígenas mayangnas ha venido cambiando lentamente, al hecho de tener escuelas de primaria unidocentes atendiendo multigrados, a escuelas de primaria regular completa multi docentes y en los últimos años las comunidades indígenas del cuarto territorio indígena (Mayangna Sauni Arunka) y la comunidad indígena de Wasakín han ampliado su cobertura educativa a secundaria completa.

Existe una motivación evidente de un grupo de jóvenes con una alta demanda por educación formal y acceso a la Educación Superior, lo que se puede reflejar en que actualmente las edades de las mujeres egresadas del bachillerado oscilan entre los 17 y 35 años de edad, en su mayoría casadas y con un promedio de 1 a 5 hijos, lo que no fue una limitante para que terminaran el bachillerato.

La mayoría de las egresadas de secundaria tuvieron aspiraciones de inscribirse en la universidad, mismas que no fueron concretadas, algunas señalan que todavía mantienen vivas sus aspiraciones, refiriendo que entre las principales dificultades para tener acceso a la Educación Superior se encuentran obstáculos económicos, culturales basados en la concepción de género, maternidad y división sexual del trabajo, la autoridad paterna y materna que enfrentan, y en menos proporción el idioma.

\section{Obstáculos económicos}

Históricamente se ha manejado que los pueblos indígenas son muy pobres, pobreza que proviene de la situación de colonización y subordinación forzosa a que los ha sometido la sociedad y los Estados en la mayoría de los casos, lo que ha limitado y continua limitando a las mujeres indígenas el acceso y permanencia a la Educación Superior. 
En este marco de escasez de recursos los roles tradicionales de género hacen que las mujeres (hijas, hermanas, esposas) tengan menos acceso a recursos económicos que los hombres. En este sentido se puede señalar el escaso nivel de control que ellas tienen sobre los recursos familiares y la participación en la toma de decisiones de los gastos, la postergación de las necesidades de las mujeres en la distribución de los recursos económicos al interior de la familia, la resistencia a la posibilidad que las mujeres trabajen de manera remunerada, porque tienen que resolver las necesidades de la esfera reproductiva en el espacio doméstico y privado, por otro lado está la escasez de fuentes de trabajo remunerado para mujeres indígenas, lo que contribuye a limitar su autonomía económica.

Adicionalmente la pobreza influye para reducir las posibilidades de las jóvenes indígenas de acceder a la Educación Superior, porque en muchas ocasiones las familias no pueden darse el lujo de perder a un integrante de su fuerza de trabajo. Esta situación además de impedir el acceso también ha traído consigo la deserción universitaria de la mayoría de mujeres indígenas que permanecían en el programa de becas de la URACCAN.

\section{Obstáculos culturales basados en concepciones de género}

El aspecto cultural es una de las áreas donde las mujeres encuentran mayores limitaciones para acceder a la educación, para muchas de las mujeres estar casadas significa asumir roles del espacio privado en lo reproductivo, mismos que han sido tradicionalmente asignados a las mujeres. Aunque muchas señalan que sus madres y padres tienen interés que ellas puedan continuar estudiando, estos no se involucran en sus asuntos una vez que ellas están casadas, la decisión debe tomarse entre ellas y sus maridos, los que en su mayoría rehúsan aceptar que sus esposas abandonen la comunidad para ir a estudiar.

En este sentido los sesgos de género tales como el escaso nivel de autonomía que tienen en cualquier tipo de decisión respecto a su propia vida, el nivel de control de los comportamientos de las mujeres, de todas las edades y en cualquier situación familiar sigue siendo un obstáculo para el desarrollo personal de las mujeres indígenas, limitando el ejercicio pleno de su ciudadanía.

\section{Maternidad y división sexual del trabajo}

Sobre la mujer indígena, conductora de la familia, dependen también la salud, la alimentación y la educación de la niñez, esta situación es considerada exclusiva de las mujeres en el cuarto territorio indígena (Mayangna Sauni Arunka) y la comunidad Wasakín, la mayoría de las mujeres cuentan con un promedio de $1-5$ hijos e hijas, asumiendo toda la responsabilidad del cuidado y de la familia en general; refieren que para estudiar dentro de la comunidad ello no significó mayores complicaciones; sin 
embargo, salir de la comunidad implica garantizar remuneración para una persona que los cuide y sus condiciones económicas no lo permiten. Para muchas salir embarazadas al momento de egresar del bachillerato ha sido una limitante para poder aspirar a la universidad, la falta de conocimientos en salud sexual y reproductiva acompaña tal situación.

La dicotomía de los roles de género persiste en las relaciones de pareja, en este apartado se hace especial mención a una particularidad del territorio Mayangna Sauni Arunka (Cuarto territorio indígena) donde existen varios graduados universitarios y algunos que actualmente son estudiantes de la URACCAN, cuando ellos estudiaron ya eran casados y padres de familia; sin embargo, esta no fue una limitante para que ellos accedieran y permanecieran en la universidad, lo curioso del caso es que ellos no han hecho muchos esfuerzos porque sus esposas vayan a la universidad, en algunos casos la situación solamente ha quedado en promesas y planes a futuro.

Se puede apreciar muy arraigada y drásticamente conservadora la reproducción de una división sexual del trabajo y roles tradicionales de género en el que a las mujeres se les sigue asignando la responsabilidad del ámbito privado.

\section{La autoridad paterna y materna}

Por su parte las mujeres solteras, hijas de dominio como se les conoce en nuestra cultura, deben enfrentar entre otras dificultades la concepción que las mujeres cuando llegan a la universidad en su mayoría salen embarazadas, situación que se ha interiorizado tanto por padres y madres de familia como por las mismas muchachas, viendo esta situación como un problema sólo de las mayangnas.

Esta situación tiene que ver tanto con aspectos culturales como con situaciones concretas, dado que algunas jóvenes que han salido a estudiar en la universidad han regresado por quedar embarazadas, haciendo aun más difícil la situación para las jóvenes que aspira ingresar a la universidad por el temor persistente de padres y madres de familia que suceda igual con sus hijas. Las jóvenes por su parte también consideran que son un problema, igualmente las autoridades han podido identificar esta situación.

Por su parte las autoridades comunales y/o territoriales, así como también las organizaciones existentes en la comunidad no han realizado acciones concretas para preparar a los jóvenes en el tema de salud sexual y reproductiva, persistiendo aún los tabúes con relación a la temática.

También es necesario señalar que en cuanto a la maternidad, las mujeres indígenas no deciden cuándo ser madres, en la mayoría de los casos ven la maternidad como un 
hecho natural e inevitable por el hecho de ser mujer. Culturalmente el matrimonio y la maternidad es un símbolo de respeto para las mujeres.

\section{Obstáculos lingüísticos}

En última instancia señalan como un segmento importante vivir en el espacio rural y tener la lengua materna como principal lengua de comunicación, lo que representa dificultad para acceder a la universidad, afirmando que tendrían problemas para acostumbrarse a hablar español, pero que sólo sería al inicio, dificultad que consideran únicamente se supera con la práctica.

Sin embargo, adicionalmente las entrevistadas señalan que la desventaja del monolingüismo las afecta de manera diferenciada respecto a sus compañeros, porque ellas tienen menos oportunidades de contacto con hablantes de otros idiomas, ya que "no salen de la comunidad" o salen mucho menos a diferencia de sus coetáneos.

Todas estas limitaciones que han experimentado, las han vivido como parte del comportamiento natural del entorno social en el que han crecido. En lo que respecta a la visión de que "el hombre trabaja y la mujer se queda en casa", según la valoración de las entrevistadas más jóvenes consideran que poco a poco va desapareciendo para dar paso a una nueva ideología en la que también las mujeres son sujetas de derechos y deben gozar de los mismos.

Hablar de Educación Superior no sólo se trata del acceso sino también de la permanencia dentro del sistema educativo. Las mujeres indígenas mayangnas para permanecer, además de los obstáculos económicos, culturales basados en la concepción de género, maternidad y división sexual del trabajo, la autoridad paterna y materna, enfrentan otros obstáculos entre los que se destacan el tratamiento en igualdad de condiciones.

\section{Tratamiento en igualdad de condiciones}

La URACCAN ha venido debatiendo sobre acciones afirmativas en la Educación Superior, como parte del nuevo modelo de universidad comunitaria que ha puesto en el debate el cuestionamiento sobre los segmentos y sectores sociales que menos acceden a la Educación Superior, esta condición que hace necesario entender el concepto de "igualdad de oportunidades". Ante este escenario, las autoridades comunales señalan que como pueblos indígenas y en especial las mujeres, no han sido partícipes de acciones afirmativas que garanticen un mayor acceso y permanencia de las mujeres en la Educación Superior y que una vez estando dentro de la universidad esa igualdad de oportunidades les hace sentir como una más del grupo de universitarias. 
Por su parte, las autoridades de la universidad señalan que existe un programa de bienestar estudiantil aprobado en el año 2007, cuenta con el apoyo financiero de Horizont30oo, orientado hacia el acceso a la Educación Superior de jóvenes costeños y costeñas del área rural y urbana, tanto de los pueblos indígenas como mestizos. Tiene como objetivo general: Acompañar de manera sistemática el proceso de formación integral de los estudiantes de la URACCAN y como objetivos específicos: 1. Promover la permanencia y graduación de los estudiantes de la universidad 2. Brindar atención vocacional a los estudiantes de primer ingreso de la universidad 3. Brindar atención psicosocial a los estudiantes de la universidad 4. Fomentar la participación de los estudiantes en actividades extracurriculares 5. Promover actividades deportivas y culturales 6 . Fomentar actitudes de solidaridad, principios morales y éticos.

Los requisitos para acceder al programa son: deseo de superación, presentar solicitud formal ante las instancias correspondientes, intención del estudiante o la estudiante para acceder a la universidad y debe hacerse por escrito, notas de 4to y 5to año, diploma de bachiller, cédula de identidad y partida de nacimiento, carta de referencia comunitaria y record policial; estos últimos dos requisitos a solicitud del organismo donante, con la intención de garantizar la seguridad estudiantil. Estos requisitos son iguales para hombres y mujeres de las diferentes etnias del territorio.

La beca incluye alimentación, albergue, atención médica, enseres personales, exoneración de aranceles, actividades extracurriculares, participación en actividades que organiza la universidad, participación en actividades deportivas, atención psicosocial, en caso de tener 90\% de rendimiento académico se exoneran del pago de aranceles.

En el año 2009, cuatro mujeres indígenas accedieron al programa de beca siendo una de ellas del territorio Mayangna Sauni Arunka (Cuarto territorio indígena), en el año 2010 solamente ingresó al programa de becas una mujer indígena. Cabe señalar que estas mujeres se retiraron antes de concluir el primer semestre del 2010, por lo que actualmente no existe ninguna mujer mayangna en el programa de becas.

\section{La maternidad y la salud}

Según autoridades universitarias entrevistadas, la maternidad y la salud se encuentran ocupando las principales razones por las que las mujeres abandonan la universidad, en la mayoría de los casos los problemas de enfermedad casi siempre están acompañados de los embarazos, información que ha sido ratificada por las desertoras y sus madres y padres de familia. Se asegura que al momento que las mujeres abandonan la universidad se les deja muy claro que siguen manteniendo la oportunidad de regresar una vez que resuelvan sus problemas de salud u otro tipo de problemas que enfrentan y que pueden regresar cuando ellas lo estimen conveniente; empero, refieren que no 
se ha realizado un plan de seguimiento o acciones concretas que garanticen el rescate de las mujeres desertoras.

Se señala que a través del área de bienestar estudiantil se desarrollan charlas y/o talleres sobre salud sexual y reproductiva de cara a disminuir esta problemática; no obstante, los resultados en la mayoría de las mujeres indígenas mayangnas no han sido óptimos.

Realizando un análisis desde la perspectiva intercultural de género es necesario valorar si los talleres y charlas están abordando situaciones tales como: la forma en la que viven la relación con su novio, la capacidad de hacer valer sus necesidades y opciones de vida y más aun abordar el proyecto de vida de las jóvenes si lo tienen o no y qué grado de autonomía tienen, si la sexualidad y la maternidad están incluidos es su proyecto de vida, tomando en cuenta el significado que para ellas tiene.

\section{Adaptación a la vida universitaria}

Para atender los problemas de adaptación a la vida universitaria, a sus ritmos y códigos, de integración con las compañeras y compañeros universitarios indígenas y no indígenas y las dificultades de comprensión de los contenidos curriculares las autoridades afirman que se han generados procesos de trato diferenciado a las mujeres y hombres Mayangnas, acompañamiento psicosocial y académico en aquellas asignaturas que más problemas presentan, afirmación que discrepa con lo que plantean las mujeres universitarias desertoras, quienes afirman no haber recibido ningún tipo de atención especial.

Es preciso profundizar a nivel de la comunidad universitaria la significación de adaptación a la vida universitaria, lo que implica ir más allá del comportamiento académico, significa pues trastocar aspectos de la cultura y la cosmovivencia de los pueblos indígenas, se hace especial mención a algunos problemas que surgen en los internados, como son los robos; más que entenderlo como robo es preciso hacer otro tipo de lectura la que puede iniciar por preguntarse ¿Por qué hacen ellas este tipo de acciones? ¿Será realmente robo lo que quieren hacer? $\mathrm{O}$ ¿Es parte de su cultura o costumbres, ponerse la ropa de sus amigas y amigos?

Por otro lado, es preciso señalar que existe voluntad expresa por parte de los docentes de acompañar a las estudiantes indígenas mayangnas en los procesos académicos; antes bien no hay una persona indígena mayangna que les atienda en su propia lengua y que se dedique de tiempo completo a este acompañamiento. 


\section{Discriminación por razones de género}

No se puede dejar de hablar de la discriminación por razones de género y etnia, algunas de las mujeres desertoras de la universidad reconocen haber sido víctimas de discriminación por razones de género y etnia.

\section{Organizaciones que inciden en las comunidades indígenas en la inserción de las mujeres a la Educación Superior}

La diversidad social y cultural ha servido en la mayoría de las sociedades como sustento de la persistencia de las inequidades, de condiciones generales desfavorables, lo que ha llevado a pueblos indígenas, mestizos y afrodescendientes a diversas expresiones de organización encabezados por líderes y lideresas indígenas haciendo escuchar sus voces y demandas, articulando sus propuestas en distintos ámbitos, particularmente en referencia a la educación. En el contexto del territorio Mayangna Sauni Arunka y la comunidad de Wasakín, se han venido manteniendo estructuras organizativas tradicionales tales como Consejo de ancianos, Síndico y Whitas comunales.

En el territorio Mayangna Sauni Arunka, a raíz de contar con profesionales del territorio y de cara a fortalecer los avances organizativos, se fundó la organización MATUMBAK (organización mayangna del rio Tunki), mediante la cual líderes indígenas han logrado articular un discurso con diversas argumentaciones que, tienen que ver con la creación de condiciones para negociar sus derechos en el contexto de la globalización.

Por otro lado el territorio Mayangna Sauni Arunka cuenta con la organización de mujeres MAYAKAB (organización de mujeres del Río Tunkí), en la que convergen mujeres de todas las comunidades que integran el territorio. Esta organización es de reciente data, lo que implica que está en proceso de fortalecimiento la educación, si bien se considera de gran relevancia para el desarrollo de las mujeres, en sus planes de acción no aparece como prioridad su gestión y quehacer.

Respecto a las demandas de las organizaciones indígenas del territorio Mayangna Sauni Arunka y la comunidad Wasakín al acceso y permanencia de las mujeres en la Educación Superior se encuentra casi nula la gestión, tanto las mujeres, padres y madres de familia, así también las autoridades comunales señalan que las prioridades para las organizaciones comunales y/o territoriales son la recuperación del territorio, el control de los recursos naturales y del patrimonio cultural, el fortalecimiento y desarrollo de sus comunidades y la revalorización de su cultura. No existe aún una problematización formulada en términos colectivos para la Educación Superior, ni una agenda vinculada a la educación universitaria. 
Reconocen la existencia a nivel municipal y regional de muchas organizaciones e instituciones que enfocan su quehacer a la defensa de los derechos de las mujeres, las cuales no han hecho presencia en el territorio.

Señalan un acercamiento con la URACCAN que es la Universidad Intercultural de los Pueblos Indígenas y Comunidades Étnicas de la Costa Caribe nicaragüense, que contribuye a fortalecer la Autonomía Regional a través del acompañamiento a los procesos de autogestión, unidad multiétnica y la formación integral de mujeres y hombres costeños.

Este acercamiento lo han asumido a través de las visitas de la universidad para ofertar su programa académico y de beca, visitas que las han realizado a través del personal de la universidad, también se han aprovechado en el caso de Wasakín el trabajo de extensión comunitaria y social que realiza la universidad desde los diferentes institutos y centros.

Otra forma de conocer la oferta académica de URACCAN ha sido a través de la radio y la información que pueden brindar los estudiantes de la comunidad que están estudiando o estudiaron en la universidad.

\section{Acciones que impulsen a las mujeres para acceder y permanecer en la Educación Superior}

La universidad comunitaria intercultural conforma un modelo de educación incluyente, que tiene como propósito formar profesionales e intelectuales comprometidos con sus culturas, región y comunidades. Ante el limitado acceso y permanencia de las mujeres indígenas a la Educación Superior, pueden sugerirse al menos tres acciones que en su momento pueden formar estrategias para mejorar el acceso y la permanencia de las mujeres indígenas a la Educación Superior:

La primera es mantener el otorgamiento de becas a estudiantes indígenas priorizando a las mujeres, bajo los parámetros que se explican en el resultado dos; pero aquí se señala que en determinado momento este programa de beca puede atravesar una difícil situación dada la vulnerabilidad que implica depender de la cooperación externa.

En este contexto sería preciso retomar la iniciativa de Chile donde se cuenta con un Programa Nacional de Becas Indígenas desde el año 1991 financiado en su totalidad por el Estado. En este sentido las autoridades comunitarias con la ayuda metodológica de la URACCAN pueden empezar el proceso de formulación de la propuesta, la cual sería gestionada por las vías correspondientes en la demanda al reconocimiento de la multietnicidad de las regiones autónomas y el nivel de vida de las mismas, así como también puede ser un modelo de política compensatoria a las inequidades existentes. 
La segunda, mantener los apoyos tutoriales para que las estudiantes indígenas cuenten con el soporte académico que les permita superar sus deficiencias educativas y nivelar sus conocimientos con relación al resto de estudiantes y que además incorpore elementos de la cosmovivencia de las mujeres indígenas. Esto como parte de la pertinencia y la responsabilidad social que debe caracteriza a las instituciones de Educación Superior, dada la necesidad de verse reconocidos y de socializar con aquellos que son originarios de su mismo grupo cultural o de otros distintos, les permitirá reivindicar su origen cultural frente a aquellos que antes los intentaban marginar.

La tercera, el diagnóstico educativo en el que se priorice el análisis de factores socioculturales e ideológicos como determinantes de la situación de la mujer indígena, lo que permitirá entender su comportamiento y además partir de las necesidades.

Las investigaciones y/o diagnósticos deben ser acompañados de un programa de capacitación sobre género con la participación de las mujeres, adolescentes, jóvenes y adultas, como un aporte al cambio de los estereotipos de género, teniendo en cuenta la cosmovivencia cultural.

Batallar con las inequidades de género arraigadas en nuestra cultura implica hacer un trabajo desde la Educación Primaria y Secundaria, he aquí un punto a favor de la universidad, dado que se cuenta con el Subsistema de Educación Autonómico Regional (SEAR) que favorece la educación con enfoque intercultural de género, por lo que la universidad debe apuntar hacia el seguimiento de la aplicación del mismo garantizando así el empoderamiento de las mujeres sobre sus derechos y el manejo de la salud sexual reproductiva, la extensión comunitaria es una de los mecanismos que puede facilitar esta labor.

\section{Conclusiones}

La diferencia cultural y la pobreza que implica la falta de recursos monetarios en efectivo, han sido los principales obstáculos que limitan a las jóvenes indígenas al acceso y permanencia en la Educación Superior.

Las mujeres indígenas no tienen la posibilidad de ejercer plenamente sus derechos ciudadanos en igualdad de condiciones, si carecen de oportunidades para desarrollar sus capacidades y talentos.

El interés expreso de las mujeres por elevar su nivel académico se ve obstaculizado por la reproducción de roles tradicionales de género, tal es el caso de los matrimonios a temprana edad y la maternidad lo que limitan a la mujer a permanecer en el ámbito privado asumiendo la responsabilidad del cuido de los hijos y de la familia en general. 
Para las madres y padres de familia la prioridad en la educación siguen siendo los hijos varones, aunque refieran consideraciones de importancia a la educación en la vida de las mujeres. Los embarazos han sido la causa principal por la que la mayoría de las mujeres indígenas han abandonado la universidad, encontrando en algunos casos la posibilidad de volver a reincorporarse si y solo sí cuentan con el apoyo de sus padres y madres.

No existe aún una problematización formulada en términos colectivos para la Educación Superior, ni una agenda vinculada a la educación universitaria por parte de las organizaciones comunitarias.

De las organizaciones e instituciones municipales y/o regionales solamente la URACCAN hace presencia en el territorio Mayangna Sauni Arunka y la comunidad Wasakín para promover el acceso y la permanencia de las mujeres en la Educación Superior garantizando un sistema de becas.

Existe la necesidad de mejorar la Educación Intercultural Bilingüe en la Educación Primaria y Secundaria para eliminar la desventaja del idioma para mujeres y hombres mayangnas, pero en especial para las mujeres que tienen un nivel de competencia lingüística en L2 inferior a los hombres.

\section{Lista de referencias}

Alemán, C. (s/f). Sistema Educativo Autonómico Regional. Instituto de Promoción e Investigación Lingüística y Rescate Cultural (IPILC) URACCAN.

Callamard, A. (2002). Metodología de investigación con enfoque de género y sensible a las mujeres indígenas. Centro internacional de derechos humanaos y desarrollo democrático.

Castillo, L. , Lee. B. (200). Impacto del proceso educacional en los graduados y graduadas, el caso de la carrera de Ingeniería Agroforestal del Recinto URACCAN Las Minas, Generación 1998-2002. Universidad de las Regiones Autónomas de la Costa Caribe Nicaragüense.

López, L. (2009). Interculturalidad, educación y ciudadanía. Perspectivas latinoamericanas. Bolivia, FUNPROEIB Andes.

Luke, C. (1999). Feminismos y pedagogías en la vida cotidiana. España, Ediciones Morata, S. L.

Saballos, JL. (2007). Manual de educación ciudadana intercultural y autonómica, Módulo I Construyendo conceptos. URACCAN, Managua Nicaragua. 
URACCAN (2004). Modelo Pedagógico de URACCAN. Aprobado en sesión ordinaria 03 - 2004 del Concejo Universitario de URACCAN, Managua o6 al o8 de Septiembre. Nicaragua.

Bonder G. (1994) "Mujer y Educación en América Latina: hacia la igualdad de oportunidades". Recuperado de http: //www. rieori. org, el 15 de Diciembre 2009.

Feito, R. (2009). Teorías sociológicas de la educación. Recuperado de http: //www. ucm. es/BUCM/cps/lecturas/4. htm, el 12 de Diciembre 2009.

Madrigal, R. , et al. (2005). Barreras y limitaciones de la mujer para su desarrollo: "Caso de la mujer estudiante”. Recuperado de http: //www. dtp. uem. br, el o4 de Abril 2010.

Pedroza, R. Villalobos, G (2008). Políticas compensatorias para la equidad en la educación superior en Ecuador y Perú. Recuperado de www. caei. com. ar, el o4 de Abril 2010.

Reynaga, G. (2008). "Mujeres Indígenas y la Educación Superior en el Perú". Recuperado de http: //ISEES. fundacionequitas. org, el 12 de Diciembre 2009.

Velázquez, B. (2006). Educación y mujeres indígenas. Recuperado de www. xiranhua. com, el 20 de Noviembre 2009. 